\title{
Heavier alkali-metal gallates as platforms for accessing functionalized abnormal NHC carbene-gallium complexes
}

\author{
Marina Uzelac, ${ }^{\text {[a] }}$ Alan R. Kennedy, ${ }^{[a]}$ Alberto Hernán-Gómez, ${ }^{[a]}$ M. Ángeles Fuentes ${ }^{[a]}$ and Eva \\ Hevia*[a]
}

Abstract: By sequentially treating the unsaturated carbene $\operatorname{IPr}(\mathrm{IPr}=$ 1,3-bis-(2,6-diisopropylphenyl)imidazol-2-ylidene) with heavier alkalimetal alkyls $\mathrm{NaR}$ or $\mathrm{KR}\left(\mathrm{R}=\mathrm{CH}_{2} \mathrm{SiMe}_{3}\right)$ and $\mathrm{GaR}_{3}$, novel heteroleptic gallates $\mathbf{1}$ and $\mathbf{2}$ have been prepared. Incorporating anionic NHC ligands, these bimetallic complexes react selectively with electrophilles to afford neutral abnormal $\mathrm{NHC}$ Ga complexes under mild conditions.

$\mathrm{N}$-heterocyclic carbenes (NHCs), particularly imidazol-2-ylidenes, have played a pivotal role in advancing key areas of modern chemistry including transition-metal catalysis, ${ }^{[1]}$ stabilisation of low valent main-group compounds ${ }^{[2]}$ and the development of frustrated Lewis pair systems, ${ }^{[3]}$ to name just a few. By modifying the substituents on the $\mathrm{N}$ atoms or at the backbone of the imidazole ring, the electronic and steric properties of NHCs can be finely tune, making these commodity ligands extremely versatile. ${ }^{[4,5]}$ Typically imidazol-2-ylidenes bind to metal fragments through their $\mathrm{C} 2$ (normal) position although in some cases coordination can occur via a carbon from the imidazole backbone (C4 or abnormal coordination)..$^{[4,5]} \mathrm{NHCs}$ can also exist as anionic moieties, as the result of their formal deprotonation, acting as a bridge between two metal centres employing simultaneously their $\mathrm{C} 2$ and $\mathrm{C} 4$ positions. ${ }^{[6]}$ Interestingly, Robinson has shown that electrophilic interception of anionic $\mathrm{NHC}$ complexes with $\mathrm{MeOTf}$ or $\mathrm{HCl} \cdot \mathrm{NEt}_{3}$ can be employed to prepare abnormal NHC complexes (aNHCs) of $\mathrm{B}$ and $\mathrm{Zn}{ }^{[7]}$ More recently our group has also used a similar approach for the synthesis of aNHC complexes of $\mathrm{Ga}$ and $\mathrm{Fe}$, where the normal $\mathrm{C} 2$ position of the carbene is blocked by a methyl group. ${ }^{[8,9]}$ Carbanionic NHCs can be prepared by several methods including chemical reduction and metal-mediated $\mathrm{C}-\mathrm{H}$ activation, however deprotonative metallation appears to be one of the most versatile approaches. ${ }^{[10]}$

The vast majority of the studies on NHC metallation have focussed on using organolithium reagents as a base, ${ }^{[6,8]}$ although mixed-metal systems such as zincates and magnesiates have shown great promise for the zincation or magnesiation of $\quad \mathrm{Pr} \quad(\mathrm{IPr} \quad=\quad$ 1,3-bis-(2,6diisopropylphenyl)imidazol-2-ylidene). ${ }^{[11,12]}$ Extending the scope of these investigations here we explore the metallating ability of heavier alkali-metal alkyls to prepare gallate complexes containing anionic NHC fragments and their applications to access novel aNHCs $\mathrm{Ga}$ complexes with a variety of substituents.

We started our studies by employing heavier alkali-metal alkyls

[a] M. Uzelac, Dr A. R. Kennedy, Dr A. Hernán-Gómez, Dr M. Á. Fuentes, Prof E. Hevia

WestCHEM, Department of Pure and Applied Chemistry

University of Strathclyde

Glasgow, G1 1XL (UK)

E-mail: eva.hevia@strath.ac.uk, marina.uzelac@strath.ac.uk

Supporting information for this article is given via
$\mathrm{NaR}$ and $\mathrm{KR}\left(\mathrm{R}=\mathrm{CH}_{2} \mathrm{SiMe}_{3}\right)$ for direct metallation of IPr as this methodology seems to be underdeveloped. Treating a hexane suspension of $\mathrm{IPr}$ with $\mathrm{MR}(\mathrm{M}=\mathrm{Na}, \mathrm{K})$, led to the instant formation of yellow solids which were completely insoluble even when using large amounts of the more polar solvent THF. Interestingly, addition of $\mathrm{GaR}_{3}$ solubilised these products allowing the isolation of heteroleptic alkali-metal gallates (THF) ${ }_{3} \mathrm{Na}\left[: \mathrm{C}\left\{\left[\mathrm{N}\left(2,6-{ }^{-} \mathrm{Pr}_{2} \mathrm{C}_{6} \mathrm{H}_{3}\right)\right]_{2} \mathrm{CHCGa}\left(\mathrm{CH}_{2} \mathrm{SiMe}_{3}\right)_{3}\right\}\right] \quad$ (1) and $(\mathrm{THF})_{3} \mathrm{~K}\left[: \mathrm{C}\left\{\left[\mathrm{N}\left(2,6-{ }^{-} \mathrm{Pr}_{2} \mathrm{C}_{6} \mathrm{H}_{3}\right)\right]_{2} \mathrm{CHCGa}\left(\mathrm{CH}_{2} \mathrm{SiMe}_{3}\right)_{3}\right\}\right]$ (2) in 71 and $76 \%$ isolated yields respectively (Scheme 1 ).

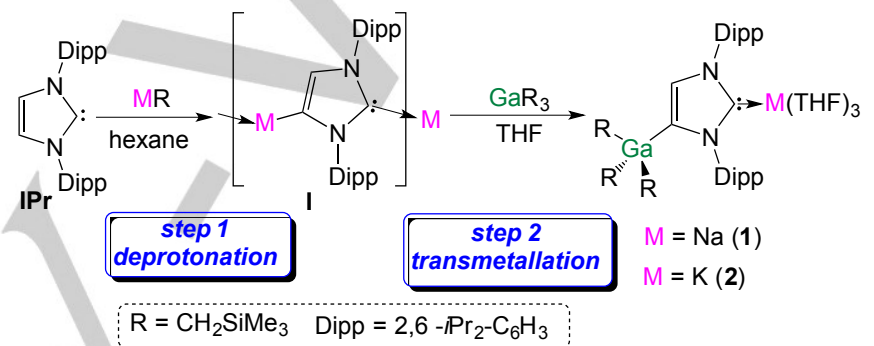

Scheme 1. Stepwise indirect gallation of IPr affording alkali-metal gallates 1 and 2.

Formation of gallates 1 and $\mathbf{2}$ can be rationalised in terms of a stepwise indirect gallation process. IPr is first deprotonated at the C4 position by the highly polar MR reagent (I in Scheme 1), which can then undergo fast transmetallation with the more electronegative $\mathrm{Ga}$ fragment, with the alkali-metal being trapped by the vacant $\mathrm{C} 2$ site of the carbene (Scheme 1). Although the solids obtained by treating IPr with MR cannot be characterised, due to their lack of solubility, the isolation of $\mathbf{1}$ and $\mathbf{2}$ provides compelling proof that these heavier alkaline metal alkys can in fact metallate this $\mathrm{NHC}$. Interestingly, when the single metal alkyl reagents are combined to form tetraorganogallates $\mathrm{MGaR}_{4},{ }^{[13]}$ the metallation process is inhibited yielding instead the coordination adducts $\left[\mathrm{IPr}_{2} \mathrm{~K}\right]\left[\mathrm{GaR}_{4}\right]$ (see SI for details) which highlights the potential of using bimetallic systems in a sequential manner. ${ }^{[14]}$ While the relevant $\mathrm{M}^{+} \mathrm{IPr}^{-}$salts (I) are obtained via direct metallation, it should be noted that Goicoechea has structurally characterised $\mathrm{K}^{+} \mathrm{IPr}^{-} \cdot 2 \mathrm{THF}$ as the result of the reaction of the lithiated $\mathrm{IPr}$ with potassium tertbutoxide. ${ }^{[15]}$

Both sodium (1) and potassium gallate (2) exhibit discrete contacted ion-pair (CIP) structures where anionic NHC ligand coordinates to the alkali metal through its normal $\mathrm{C} 2$ position while $\mathrm{Ga}$ occupies the position previously filled by a $\mathrm{H}$ atom, bonding to the $\mathrm{C} 4$ position (Fig 1). The $\mathrm{Ga}-\mathrm{C} 4$ distances (i.e. C24 for 1 and C2 for 2 in Fig 1) of 2.050(2) A for 1 and 2.050(3) $\AA$ for 2 are close in value with the Ga- $C_{\text {alkyl }}$ bonds (average $2.027 \AA$ and $2.028 \AA$ for 1 and 2 respectively) and they are also in excellent agreement with the bond distances found in the previously reported lithium congener. ${ }^{[8]}$ The narrow variation observed for the Ga-C bond lengths together with very similar 
bond angles [mean angle $108.15^{\circ}$ for 1 and $109.46^{\circ}$ for 2] reveal an almost ideal tetrahedral geometry of gallium centre in both compounds. With the virtually identical environment around Gaatom, complexes $\mathbf{1}$ and $\mathbf{2}$ display their differences at the other end of the bridging ligand. Unsurprisingly, the $\mathrm{M}-\mathrm{C}_{\mathrm{NHC}}$ bond distance found in 1 [2.530(3) $\AA$ ] is significantly shorter than that of $2[2.902(3) \AA]$, which is in agreement with the increase in size of the alkali-metal. Both values compare well with those reported for other anionic complexes containing these alkali-metals. ${ }^{[9,}$ $11,13,16,17]$ Both sodium and potassium complete their coordination spheres by coordination of three molecules of THF, with more electropositive potassium gaining further stabilisation through electrostatic interaction with the ipso carbon of the pendant Dipp group on N2 (Figure 1). This secondary contact $[\mathrm{K} 1 \cdots \mathrm{C} 16=$ $3.301(3) \AA]$ is within the range of previously reported potassium $\pi$-interactions ${ }^{[18]}$ and translates into a significantly more acute N2-C1-K1 angle $\left(107.77(18)^{\circ}\right)$ than N1-C1-K1 $\left(150.71(19)^{\circ}\right)$.

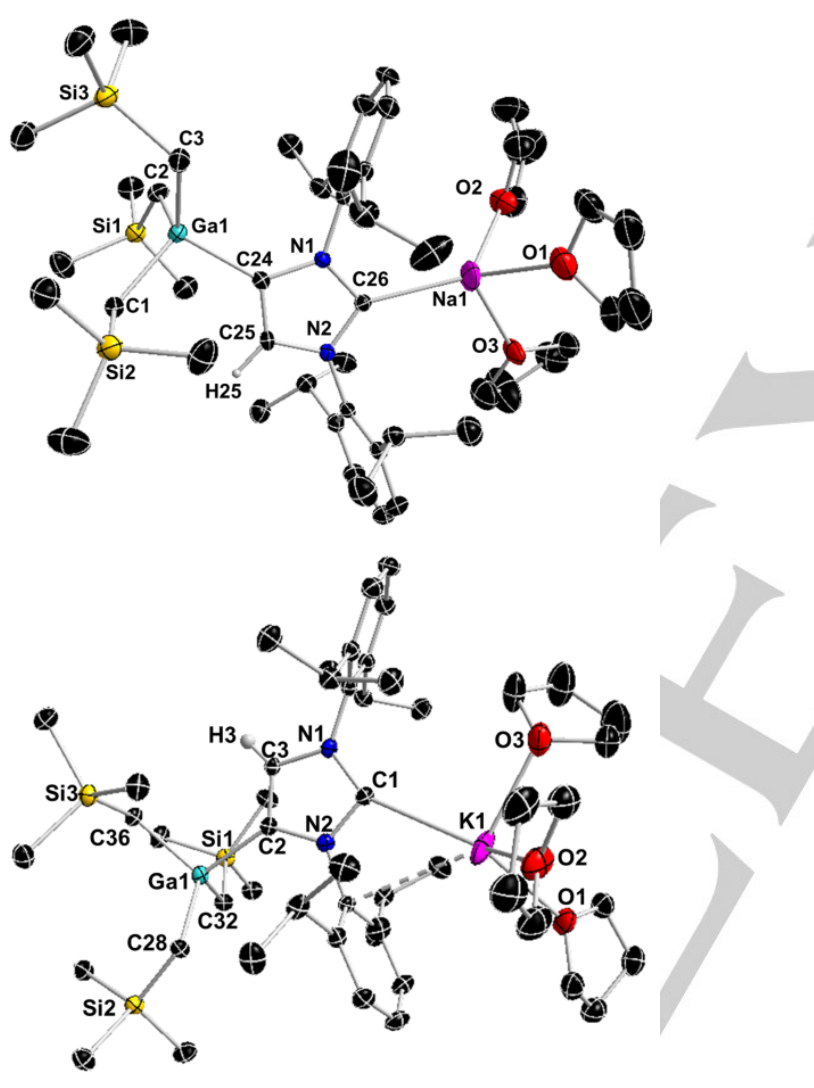

Figure 1. Molecular structure of 1 (top) and 2 (bottom) with $50 \%$ probability displacement ellipsoids. All hydrogen atoms except the one left on imidazole ring and disorder components in THF ligands have been omitted for clarity. Dashed lines represent secondary interactions.

From the NMR data of $\mathbf{1}$ and $\mathbf{2}$ in $\mathrm{d}_{8}$-THF solutions, the metallation of IPr is evident by the large downfield shift of the C4 resonance in the ${ }^{13} \mathrm{C}$ NMR spectra (from 122.3 in free IPr to 155.2 and $153.6 \mathrm{ppm}$ respectively) as well as the presence of a diagnostic singlet integrating $1 \mathrm{H}$ in the ${ }^{1} \mathrm{H}$ NMR spectra for the imidazole $\mathrm{CH}$ (at 6.64 and 6.59 ppm respectively versus 7.19 in free $\mathrm{IPr}$ ). The loss of symmetry in the imidazole ring is reflected in the ${ }^{1} \mathrm{H}$ and ${ }^{13} \mathrm{C}$ NMR spectra with the appearance of two distinct sets of Dipp signals. In addition, the carbenic $\mathrm{C}$ atom attached to the alkali-metal can be observed at 202.8 and 210.7 ppm for 1 and 2 respectively, at similar values to those reported for other related complexes containing $\mathrm{Na}^{[11]}$ and $\mathrm{K} \cdot{ }^{[15]}$ The wellestablished notion that the size and the substitution pattern of the heterocyclic ring frame can have a large effect on the properties of carbene, ${ }^{[18,19]}$ prompted us to investigate the potential of $\mathbf{1}$ and $\mathbf{2}$ as molecular synthons. To this end, $\mathbf{1}$ was reacted with an equimolar amount of allyl bromide and 2 with $\mathrm{Me}_{3} \mathrm{SiCl}$ in $\mathrm{THF}$ at room temperature. In both cases the reaction proceeded with the formation of white precipitate (presumably alkali metal salts $\mathrm{NaBr}$ and $\mathrm{KCl}$, respectively) affording $\left[\mathrm{C}_{3} \mathrm{H}_{5} \mathrm{C}\left\{\left[\mathrm{N}\left(2,6-\mathrm{Pr}_{2} \mathrm{C}_{6} \mathrm{H}_{3}\right)\right]_{2} \mathrm{CHCGa}\left(\mathrm{CH}_{2} \mathrm{SiMe}_{3}\right)_{3}\right\}\right]$ $\left[\mathrm{Me}_{3} \mathrm{SiC}\left\{\left[\mathrm{N}\left(2,6-\mathrm{Pr}_{2} \mathrm{C}_{6} \mathrm{H}_{3}\right)\right]_{2} \mathrm{CHCGa}\left(\mathrm{CH}_{2} \mathrm{SiMe}_{3}\right)_{3}\right\}\right]$ (4) in 42 and $61 \%$ yields respectively (Scheme 2). Compounds 3 and 4 are neutral abnormal NHC Ga complexes obtained as a result of the selective allylation (for 3 ) and silylation (for 4 ) of the $\mathrm{C} 2$ position of the anionic NHC ligand present in $\mathbf{1}$ and $\mathbf{2}$, leaving the C4-Ga left intact. The isolation of $\mathbf{4}$ contrasts with the reactivity reported by Arnold for a related mixed $K / Y$ complex, ${ }^{[16]}$ where the silylation occurs at the $\mathrm{C} 4$ position of the anionic carbene, instead of $\mathrm{C} 2$. Similar regioselectivity has been witnessed by Robinson for polymeric $\mathrm{Li}^{+} \mathrm{IPr}^{-}$, which in this case affords the C4$\mathrm{SiMe}_{3}$ substituted free carbene. ${ }^{[6]}$ Interestingly, by adding borane to this lithium complex, it is possible to direct the selectivity of the quench with $\mathrm{SiMe}_{3} \mathrm{Cl}$ towards $\mathrm{C}_{2} .^{[7 \mathrm{~b}]}$

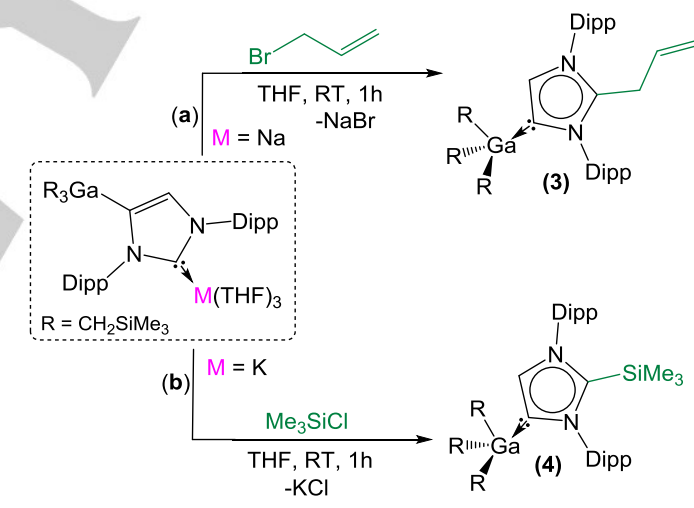

Scheme 2. Electrophilic interception of anionic NHC complexes (a) 1 with allyl bromide and (b) 2 with $\mathrm{Me}_{3} \mathrm{SiCl}$.

The molecular structures of $\mathbf{3}$ and $\mathbf{4}$ have been established by $\mathrm{X}$ ray crystallography (Fig 2). The Ga-C4 distances (i.e. C3 and C45 for 3 and 4 respectively) showed very little variation $[2.0802(18)$ and $2.0887(16) \AA]$ to that found in $\left[a \mid P r \cdot G a R_{3}\right]^{[8]}$ $(2.0759(16) \AA)$ where the $\mathrm{C} 2$ position of the carbene is occupied by a $\mathrm{H}$ atom, suggesting that the substituents on the $\mathrm{C} 2$ of the imidazole ring have little influence in the strength of the $\mathrm{Ga}-\mathrm{C} 4$ bond. Another interesting trend is that despite the neutral constitution of these novel abnormal NHCs, the $\mathrm{Ga}-\mathrm{C}$ bond distances are only slightly elongated to those discussed for the anionic precursors 1 and $\mathbf{2}$. Structural analysis of $\mathbf{3}$ revealed it to be a cocrystal which contains $\mathrm{CH}_{2}-\mathrm{CH}=\mathrm{CH}_{2}$ and $\mathrm{CH}=\mathrm{CH}-\mathrm{CH}_{3}$ as substituents at the $\mathrm{C} 2$ of the carbene in approximately $2: 3$ ratio, arising from the partial allylic rearrangement. Mirroring this 
composition in the solution, NMR spectroscopic analysis of $\mathbf{3}$ proved to be extremely complex in the allylic section (from 3 to 6 ppm); although it should be noted that no interconversion between these two isomers is observed over prolonged periods of time. Despite its complexity, the ${ }^{1} \mathrm{H}$ NMR spectrum displays four septets for the $\mathrm{CH}$ of isopropyl groups, while an informative resonance at $163.4 \mathrm{ppm}$ is observed for the carbenic carbon. A similar chemical shift was observed for 4 (167.6 ppm) along with another signal at $148.8 \mathrm{ppm}$, which can be assigned to the $C$ of the imidazole ring that is now bonded to a $\mathrm{SiMe}_{3}$ group.
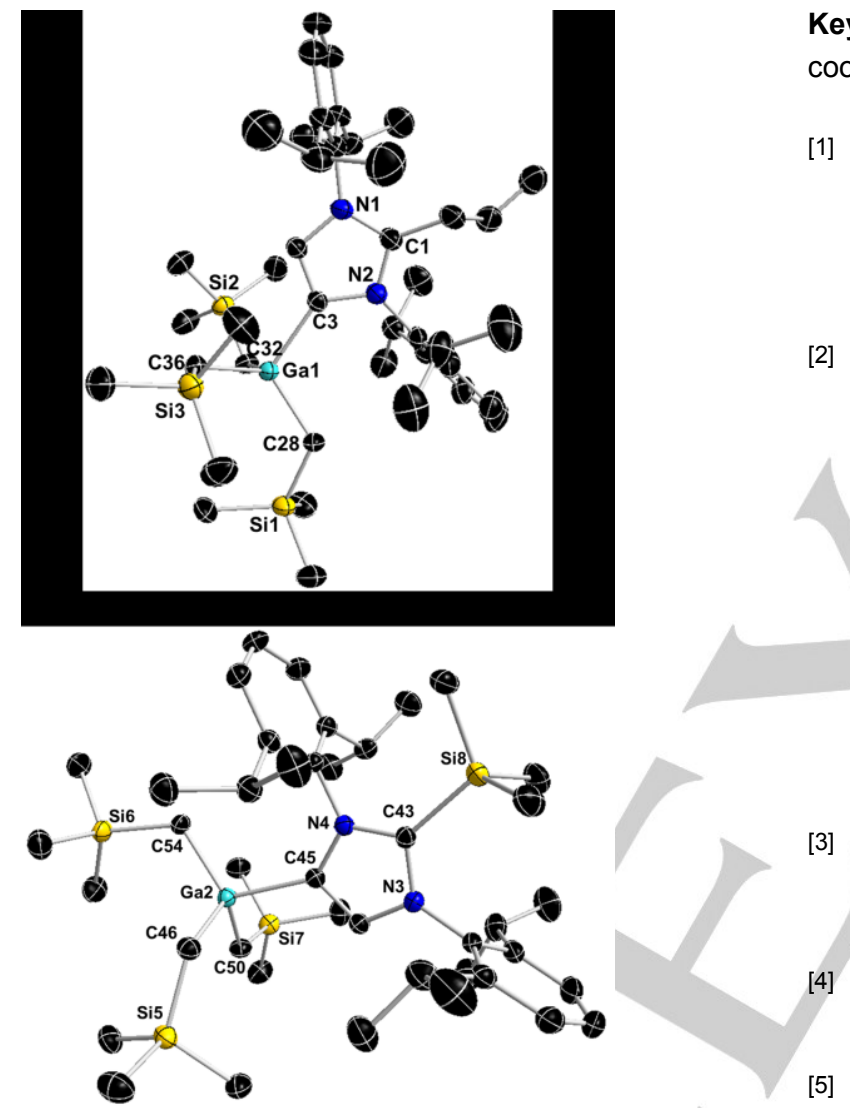

Figure 2. Molecular structure of 3 (top) and 4 (bottom) with $50 \%$ probability displacement ellipsoids. All hydrogen atoms have been omitted for clarity. For compound 3 only the $\mathrm{CH} 2-\mathrm{CH}=\mathrm{CH} 2$ fragment is shown. The unit cell of 4 contains three crystallographically independent molecules with identical connectivity. One of these molecules is shown here.

Collectively these findings show the ability of heavier alkali metal alkyls to effectively metallate unsaturated $\mathrm{NHC} \mathrm{IPr}$ when operating in tandem with a gallium alkyl, affording sodium and potassium gallate complexes 1 and 2 respectively. Electrophilic interception of these bimetallic compounds selectively yielded neutral abnormal $\mathrm{Ga} \mathrm{NHC}$ complexes disclosing the preference of anionic ligand present in $\mathbf{1}$ and $\mathbf{2}$ to react with electrophiles via its $\mathrm{C} 2$ position while preserving the $\mathrm{Ga}-\mathrm{C} 4$ bond.

\section{Experimental Section}

Full experimental details and copies of NMR spectra are included in the Supporting Information. CCDC 1501619-1501622 contain the supplementary crystallographic data of this paper. These data can be obtained free of charge from the Cambridge Crystallographic Data Centre via www.ccdc.cam.ac.uk/data request/cif.

\section{Acknowledgements}

We thank the European Research Council (ERC) for their generous sponsorship of this research.

Keywords: gallium • sodium • potassium • carbene ligands • coordination chemistry

[1] a) M. Scholl, S. Ding, C. W. Lee, R. H. Grubbs, Org. Lett. 1999, 1, 953 956; b) C. Valente, S. Calimsiz, K. H. Hoi, D. Mallik, M. Sayah, M. G. Organ, Angew. Chem. Int. Ed. 2012, 51, 3314-3332; Angew. Chem. 2012, 124, 3370-3388; c) S. Diez-Gonzalez, N. Marion, S. P. Nolan, Chem. Rev. 2009, 109, 3612-3676; d) D. Enders, O. Niemeier, A Henseler, Chem. Rev. 2007, 107, 5606-5655.

[2] a) Y. Wang, Y. Xie, P. Wei, R. B. King, H. F. Schaefer III, P. v. R. Schleyer, G. H. Robinson, Science, 2008, 321, 1069-1071; b) A. Sidiropoulos, C. Jones, A. Stasch, S. Klein, G. Frenking, Angew. Chem. Int. Ed. 2009, 48, 9701-9704; Angew. Chem. 2009, 121, 9881-9884; c) R. S. Ghadwal, H. W. Roesky, S. Merkel, J. Henn, D. Stalke, Angew. Chem. Int. Ed. 2009, 48, 5683-5686; Angew. Chem. 2009, 121, 57935796; d) C. Jones, A. Sidiropoulos, N. Holzmann, G. Frenking, A Stasch, Chem. Commun. 2012, 48, 9855-9857; e) Y. Wang, Y. Xie, P. Wei, R. B. King, H. F. Schaefer III, P. v. R. Schleyer, G. H. Robinson, J. Am. Chem. Soc. 2008, 130, 14970-14971; f) M. Y. Abraham, Y. Wang Y. Xie, P. Wei, H. F. Schaefer III, P. v. R. Schleyer, G. H. Robinson, Chem. Eur. J. 2010, 16, 432-435; g) H. Braunschweig, R. D. Dewhurst, K. Hammond, J. Mies, K. Radacki, A. Vargas, Science 2012, 336, 1420-1422.

[3] a) L. J. Hounjet, D. W. Stephan, Org. Process Res. Dev. 2014, 18, 385 391; b) D. W. Stephan, Acc. Chem. Res. 2015, 48, 306-316; c) M. Tamm in Frustrated Lewis Pair I: Uncovering and Understanding (Eds.: G. Erker, D. W. Stephan), Springer, 2013, pp 121-155.

[4] a) M. Albrecht, Chem. Commun. 2008, 3601-3610; b) O. Schuster, L. Yang, H. Raubenheimer, M. Albrecht, Chem. Rev. 2009, 109, 34453478; c) M. Albrecht, Science 2009, 326, 532-533.

[5] R. H. Crabtree, Coord. Chem. Rev. 2013, 257, 755-766.

[6] Y. Wang, Y. Xie, M. Y. Abraham, P. Wei, H. F. Schaefer III, P. v. R. Schleyer, G. H. Robinson, J. Am. Chem. Soc. 2010, 132, 14370-14372.

[7] a) Y. Wang, Y. Xie, M. Y. Abraham, R. G. Gilliard Jr., P. Wei, C. F. Campana, H. F. Schaefer III, P. v. R. Schleyer, G. H. Robinson, Angew. Chem. Int. Ed. 2012, 51, 10173-10176; Angew. Chem. 2012, 124 10320-10323; b) Y. Wang, M. Y. Abraham, R. G. Gilliard Jr., P. Wei, J. C. Smith, G. H. Robinson, Organometallics 2012, 31, 791-793; c) M. Chen, Y. Wang, R. J. Gilliard Jr., P. Wei, N. A. Schwartz, G. H. Robinson, Dalton Trans. 2014, 43, 14211-14214.

[8] M. Uzelac, A. Hernán-Gómez, D. R. Armstrong, A. R. Kennedy, E. Hevia, Chem. Sci. 2015, 6, 5719-5728.

[9] L. C. H. Maddock, T. Cadenbach, A. R. Kennedy, I. Borilovic, G. Aromí, E. Hevia, Inorg. Chem. 2015, 54, 9201-9210.

[10] J. B. Waters, J. M. Goicoechea, Coord. Chem. Rev. 2015, 293-294, 80 94.

[11] D. R. Armstrong, S. E. Baillie, V. L. Blair, N. G. Chabloz, J. Diez, J. Garcia-Alvarez, A. R. Kennedy, S. D. Robertson, E. Hevia, Chem. Sci. 2013, 4, 4259-4266.

[12] A. J. Martínez-Martínez, M. Ángeles Fuentes, A. Hernán-Gómez, E Hevia, A. R. Kennedy, R. E. Mulvey, C. T. O'Hara, Angew. Chem. Int Ed. 2015, 54, 14075-14079; Angew. Chem. 2015, 127, 14281-14285.

[13] D. R. Armstrong, E. Brammer, T. Cadenbach, E. Hevia, A. R. Kennedy, Organometallics 2013, 32, 480-489. 
[14] a) D. R. Armstrong, E. Crosbie, E. Hevia, R. E. Mulvey, D. L. Ramsay, S. D. Robertson, Chem. Sci. 2014, 5, 3031-3045; b) W. Clegg, E. Crosbie, S. H. Dale-Black, E. Hevia, G. Honeymann, A. R. Kennedy, R. E. Mulvey, D. L. Ramsay, S. D. Robertson, Organometallics 2015, 34 2580-2589.

[15] J. B. Waters, J. M. Goicoechea, Dalton. Trans. 2014, 43, 14239-14248.

[16] P. L. Arnold, S. T. Liddle, Organometallics 2006, 25, 1485-1491.

[17] P. K. Majhi, G. Schnakenburg, Z. Kelemen, L. Nyulaszi, D. P. Gates, R. Streubel, Angew. Chem. Int. Ed. 2013, 52, 10080-10083; Angew. Chem. 2013, 125, 10264-10267.

[18] a) F. Feil, S. Harder, Organometallics 2000, 19, 5010-5015; b) G. C Forbes, A. R. Kennedy, R. E. Mulvey, B. A. Roberts, R. B. Rowling, Organometallics 2002, 21, 5115-5121; c) K. L. Hull, I. Carmichael, B. C.
Noll, K. W. Henderson, Chem. Eur. J. 2008, 14, 3939-3953; d) M Uzelac, I. Borilovic, M. Amores, T. Cadenbach, A. R. Kennedy, G Aromí, E. Hevia, Chem. Eur. J. 2016, 22, 4843-4854; d) M. S. Hill, G. Kociok-Köhn, D. J. MacDougall, Inorg. Chem. 2011, 50, 5234-5241; e) M. I. Lipschutz, T. Chantarojsiri, Y. Dong, T. D. Tilley, J. Am. Chem. Soc. 2015, 137, 6336-6372.

[19] M. N. Hopkinson, C. Richter, M. Schedler, F. Glorious, Nature, 2014 510, 485-496

[20] D. Mendoza-Espinosa, B. Donnadieu, G. Bertrand, J. Am. Chem. Soc 2010, 132, 7264-7265

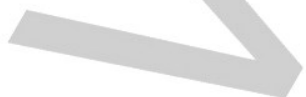


Entry for the Table of Contents (Please choose one layout)

Layout 2:

\section{COMMUNICATION}

$$
\text { iPr }
$$

Electrophilic interception of alkali-metal gallates, obtained using by indirect gallation of IPr has revealed a versatile method to access C2-substituted abnormal NHC Ga complexes
M. Uzelac, * A. R. Kennedy, A. HernánGómez, M. Á. Fuentes, E. Hevia*

Page No. - Page No.

Heavier alkali-metal gallates as platforms for accessing functionalised abnormal NHC-gallium complexes 
Additional Author information for the electronic version of the article.

$\begin{array}{ll}\text { Author: } & \text { ORCID identifier } \\ \text { Author: } & \text { ORCID identifier }\end{array}$

Author: ORCID identifier 
Z. Anorg. Allg. Chem. 2016 • ISSN 0044-2313

\section{SUPPORTING INFORMATION}

Title: Heavier Alkali-metal Gallates as Platforms for Accessing Functionalized Abnormal NHC CarbeneGallium Complexes

Author(s): M. Uzelac, * A. R. Kennedy, A. Hernán-Gómez, M. Á. Fuentes, E. Hevia* Ref. No.: Z.201600310 
Heavier alkali-metal NHC gallates as platforms for accessing functionalized abnormal carbene-gallium complexes

Marina Uzelac, ${ }^{*[a]}$ Alan R. Kennedy,${ }^{[a]}$ Alberto Hernán-Gómez, ${ }^{[a]}$ M. Ángeles Fuentes ${ }^{[a]}$ and Eva Hevia*a] $^{\text {[a] }}$

WestCHEM, Department of Pure and Applied Chemistry, University of Strathclyde, 295 Cathedral Street, Glasgow, G1 1XL, UK

eva.hevia@strath.ac.uk

marina.uzelac@strath.ac.uk

\section{Contents}

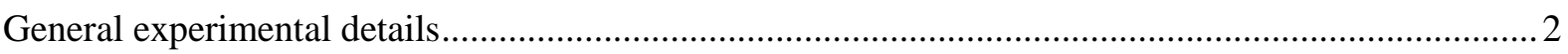

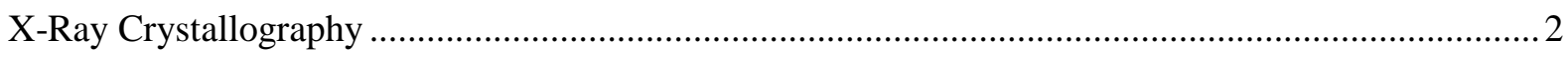

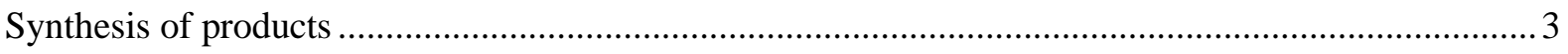








\section{General experimental details}

All reactions were carried out using standard Schlenk and glove box techniques under an inert atmosphere of argon. Solvents (THF, hexane, benzene and toluene) were dried by heating to reflux over sodium benzophenone ketyl and distilled under nitrogen prior to use. NMR spectra were recorded on a Bruker DPX $400 \mathrm{MHz}$ spectrometer, operating at $400.13 \mathrm{MHz}$ for ${ }^{1} \mathrm{H}$, and $100.62 \mathrm{MHz}$ for ${ }^{13} \mathrm{C}\left\{{ }^{1} \mathrm{H}\right\}$. Elemental analyses were obtained using a Perkin Elmer 2400 elemental analyser. Allyl bromide was purchased from Sigma Aldrich Chemicals and used as received. $\mathrm{Me}_{3} \mathrm{SiCl}$ was purchased from Sigma Aldrich Chemicals, dried over calcium hydride and stored over $4 \AA$ molecular sieves prior to use. $\left[\mathrm{Ga}\left(\mathrm{CH}_{2} \mathrm{SiMe}_{3}\right)_{3}\right],{ }^{1}\left[\mathrm{NaCH}_{2} \mathrm{SiMe}_{3}\right],{ }^{2}\left[\mathrm{KCH}_{2} \mathrm{SiMe}_{3}\right]^{2 \mathrm{a}, 3}$ and $\mathrm{IPr}^{4}$ were prepared according to literature methods.

\section{X-Ray Crystallography}

Crystallographic data for 2, 3 and $\mathbf{4}$ were measured at 123(2) K with an Oxford Diffraction Gemini S instrument with graphite-monochromated $\mathrm{Cu}(\lambda=1.54180 \AA)$ radiation. For $\mathbf{1}$ an Oxford Diffraction Xcalibur E instrument and Mo $(\lambda=0.71073 \AA)$ radiation was used. All structures were refined to convergence on $F^{2}$ using all unique reflections and programs from the SHELX family. ${ }^{5}$ The final model for all structures included constraints and restraints on bond lengths and displacement parameters that were required to model disordered groups. THF ligands (for $\mathbf{1}$ and $\mathbf{2}$ ) and three $\mathrm{SiMe}_{3}$ groups of $\mathbf{4}$ were modelled as disordered over two sites. For $\mathbf{3}$ several different models were investigated. The final model for the alkene fragment contained disordered $\mathrm{CH}_{2}-\mathrm{CH}=\mathrm{CH}_{2}(40.8 \%)$ and $\mathrm{CH}=\mathrm{CH}-\mathrm{CH}_{3}(59.2 \%)$ fragments and three separate disorder components. Selected crystallographic data are presented in Table S1 and full details in cif format can be obtained free of charge from the Cambridge Crystallographic Data Centre via www.ccdc.cam.uk/data_request/cif.

Table S1: Selected crystallographic and refinement parameters for compounds 1-4.

\begin{tabular}{|l|l|l|l|l|}
\hline Compound & \multicolumn{1}{|c|}{$\mathbf{1}$} & \multicolumn{1}{c|}{$\mathbf{2}$} & \multicolumn{1}{c|}{$\mathbf{3}$} & \multicolumn{1}{c|}{$\mathbf{4}$} \\
\hline $\begin{array}{l}\text { Empirical } \\
\text { formula }\end{array}$ & $\mathrm{C}_{51} \mathrm{H}_{92} \mathrm{GaN}_{2} \mathrm{NaO}_{3} \mathrm{Si}_{3}$ & $\mathrm{C}_{51} \mathrm{H}_{92} \mathrm{GaKN}_{2} \mathrm{O}_{3} \mathrm{Si}_{3}$ & $\mathrm{C}_{42} \mathrm{H}_{73} \mathrm{GaN}_{2} \mathrm{Si}_{3}$ & $\mathrm{C}_{42} \mathrm{H}_{77} \mathrm{GaN}_{2} \mathrm{Si}_{4}$ \\
\hline $\begin{array}{l}\text { Formula } \\
\text { weight }\end{array}$ & 958.24 & 974.35 & 760.01 & 792.13 \\
\hline Crystal system & Triclinic & Triclinic & Orthorhombic & Monoclinic \\
\hline Space group & $\mathrm{P}-1$ & $\mathrm{P}-1$ & $\mathrm{P}$ bca & $\mathrm{P} 2{ }_{1} / \mathrm{c}$ \\
\hline$\chi(\AA)$ & 0.71073 & 1.5418 & 1.5418 & 1.5418 \\
\hline$a(\AA)$ & $10.7828(8)$ & $10.8225(4)$ & $15.7786(2)$ & $16.02200(10)$ \\
\hline$b(\AA)$ & $14.5956(9)$ & $14.8126(4)$ & $18.2659(2)$ & $48.5616(3)$ \\
\hline$c(\AA)$ & $19.1526(12)$ & $19.1585(7)$ & $31.7708(5)$ & $18.64600(10)$ \\
\hline$\alpha\left(^{\circ}\right)$ & $97.397(5)$ & $99.854(3)$ & 90 & 90 \\
\hline$\beta\left(^{\circ}\right)$ & $95.292(6)$ & $94.205(3)$ & 90 & $96.3220(10)$ \\
\hline
\end{tabular}

${ }^{1}$ L. M. Dennis, W. Patnode, J. Am. Chem. Soc. 1932, 54, 182.

2 a)A. J. Hart, D. H. O’Brien, C. R. Russell, J. Organomet Chem. 1974, 72, C19; b) W. Clegg, B. Conway, A. R. Kennedy, J. Klett, R. E. Mulvey, L. Russo, Eur. J. Inorg. Chem. 2011, 721.

${ }^{3}$ B. Conway, D. V. Graham, E. Hevia, A. R. Kennedy, J. Klett, R. E. Mulvey, Chem. Commun. 2008, 2638.

${ }^{4}$ L. Hintermann, Beilstein Journal of Organic Chemistry 2007, 3, 1.

${ }^{5}$ G. M. Sheldrick, Acta Crystallogr., 2008, A64, 112. 


\begin{tabular}{|l|l|l|l|l|}
\hline$\gamma\left({ }^{\circ}\right)$ & $105.058(6)$ & $104.535(3)$ & 90 & 90 \\
\hline$V\left(\AA^{3}\right)$ & $2861.6(3)$ & $2907.28(18)$ & $9156.7(2)$ & $14419.37(17)$ \\
\hline $\mathrm{Z}$ & 2 & 2 & 8 & 12 \\
\hline$\mu\left(\mathrm{mm}^{-1}\right)$ & 0.589 & 2.160 & 1.771 & 1.934 \\
\hline $\left.2 \theta \mathrm{max}^{\circ}\right)$ & 54.994 & 146.792 & 146.484 & 146.57 \\
\hline $\begin{array}{l}\text { Measured } \\
\text { reflections }\end{array}$ & 27405 & 22187 & 41291 & 163145 \\
\hline $\begin{array}{l}\text { Unique } \\
\text { reflections }\end{array}$ & 13103 & 11344 & 9105 & 28653 \\
\hline $\begin{array}{l}\text { Observed } \\
\text { reflections }\end{array}$ & 9034 & 9125 & 7610 & 24954 \\
\hline $\mathrm{R}_{\text {int }}$ & 0.0523 & 0.0567 & 0.0405 & 0.0345 \\
\hline $\begin{array}{l}\mathrm{R}[\text { on F, obs } \\
\text { refln only] }\end{array}$ & 0.0538 & 0.0661 & 0.0391 & 0.0411 \\
\hline $\begin{array}{l}\text { wR [on F } \mathrm{F}^{2} \text {, all } \\
\text { data] }\end{array}$ & 0.1197 & 0.1792 & 0.1064 & 0.1079 \\
\hline GoF & 1.019 & 1.027 & 1.024 & 1.029 \\
\hline $\begin{array}{l}\text { Largest diff } \\
\text { peak/hole (e } \AA^{-}\end{array}$ & $0.614 /-0.811$ & $1.504 /-0.487$ & $0.367 /-0.213$ & $0.951 /-0.313$ \\
\hline 3) & & & \\
\hline
\end{tabular}

\section{Synthesis of products}

Synthesis of (THF) $)_{3} \mathrm{Na}\left[: \mathrm{C}\left\{\left[\mathrm{N}\left(2,6-{ }^{i} \mathrm{Pr}_{2} \mathrm{C}_{6} \mathrm{H}_{3}\right)\right]_{2} \mathrm{CHCGa}\left(\mathrm{CH}_{2} \mathrm{SiMe}_{3}\right)_{3}\right\}\right](\mathbf{1})$

Equimolar amounts of $\mathrm{Na}\left(\mathrm{CH}_{2} \mathrm{SiMe}_{3}\right)(0.22 \mathrm{~g}, 2 \mathrm{mmol})$ and $\operatorname{IPr}(0.8 \mathrm{~g}, 2 \mathrm{mmol})$ were suspended in hexane $(10 \mathrm{~mL})$ and stirred for $2 \mathrm{~h}$ at room temperature. To the obtained slurry, a hexane solution of $\mathrm{Ga}\left(\mathrm{CH}_{2} \mathrm{SiMe}_{3}\right)_{3}(0.66 \mathrm{~g}, 2 \mathrm{mmol}$ in $10 \mathrm{~mL}$ hexane) was added via cannula and stirred over night at room temperature. The reaction mixture was then concentrated to approximately $5 \mathrm{~mL}$ and $1 \mathrm{~mL}$ of THF was added to afford a straw solution. Overnight storage of the solution at $-30{ }^{\circ} \mathrm{C}$ provided a batch of colourless crystals $(1.36 \mathrm{~g}, 71$ $\%)$. \%). It should be noted that two coordinated THF molecules are lost upon drying in vacuo. Anal. Calcd for $\mathrm{C}_{43} \mathrm{H}_{76} \mathrm{~N}_{2} \mathrm{Si}_{3} \mathrm{NaOGa}$ : C, 63.44; H, 9.41; N, 3.44. Found: C, 63.21; H, $9.44 ; \mathrm{N}, 3.70$.

${ }^{1} \mathbf{H}$ NMR (298 K, $\left.\mathbf{C}_{\mathbf{6}} \mathbf{D}_{6}\right) \boldsymbol{\delta}(\mathbf{p p m})-0.62\left(6 \mathrm{H}, \mathrm{s}, \mathrm{CH}_{2} \mathrm{SiMe}_{3}\right), 0.37\left(27 \mathrm{H}, \mathrm{s}, \mathrm{Si}\left(\mathrm{CH}_{3}\right)_{3}\right), 1.04$ $\left(12 \mathrm{H}, \mathrm{d}, \mathrm{CH}\left(\mathrm{CH}_{3}\right)_{2}\right), 1.31\left(20 \mathrm{H}\right.$, mult, $\left.\mathrm{CH}\left(\mathrm{CH}_{3}\right)_{2}+\mathrm{THF}\right), 1.57\left(6 \mathrm{H}, \mathrm{d}, \mathrm{CH}\left(\mathrm{CH}_{3}\right)_{2}\right), 3.00(2 \mathrm{H}$, sept, $\left.\mathrm{CH}\left(\mathrm{CH}_{3}\right)_{2}\right), 3.09$ (14H, mult, THF), $3.21\left(2 \mathrm{H}\right.$, sept, $\left.\mathrm{CH}\left(\mathrm{CH}_{3}\right)_{2}\right), 6.99(1 \mathrm{H}, \mathrm{s}$, imidazole backbone $\mathrm{CH}), 7.08(2 \mathrm{H}, p-\mathrm{CH}), 7.16-7.21\left(4 \mathrm{H}\right.$, mult, $m-\mathrm{CH}$ overlapping with $\left.\mathrm{C}_{6} \mathrm{D}_{6}\right)$. ${ }^{13} \mathbf{C}\left\{{ }^{1} \mathbf{H}\right\}$ NMR $\left(298 \mathbf{K}, \mathbf{C}_{6} \mathbf{D}_{6}\right) \boldsymbol{\delta}(\mathbf{p p m}) 0.2\left(\mathrm{CH}_{2} \mathrm{SiMe}_{3}\right), 3.8\left(\mathrm{Si}\left(\mathrm{CH}_{3}\right)_{3}\right), 23.3\left(\mathrm{CH}\left(\mathrm{CH}_{3}\right)_{2}\right)$, $24.8\left(\mathrm{CH}\left(\mathrm{CH}_{3}\right)_{2}\right), 25.3\left(\mathrm{CH}\left(\mathrm{CH}_{3}\right)_{2}\right), 25.5\left(\mathrm{CH}\left(\mathrm{CH}_{3}\right)_{2}\right), 27.9\left(\mathrm{CH}\left(\mathrm{CH}_{3}\right)_{2}\right), 28.1\left(\mathrm{CH}\left(\mathrm{CH}_{3}\right)_{2}\right)$, 
$123.4(\mathrm{Ar}-\mathrm{CH}), 123.7(\mathrm{Ar}-\mathrm{CH}), 124.5(\mathrm{Ar}-\mathrm{CH}), 128.5(\mathrm{Ar}-\mathrm{CH}), 129.2$ (imidazole backbone $C H), 139.1$ (Ar- $C$ ), 142.7 (Ar- $C$ ), 146.8 (Ar- $C$ ), 146.9 (Ar- $C), 156.0(C-\mathrm{Ga}), 198.6(C$ :).

${ }^{1} \mathbf{H}$ NMR (298 K, d d -THF) $\boldsymbol{\delta}(\mathbf{p p m})-1.18\left(6 \mathrm{H}, \mathrm{s}, \mathrm{CH}_{2} \mathrm{SiMe}_{3}\right),-0.17\left(27 \mathrm{H}, \mathrm{s}, \mathrm{Si}\left(\mathrm{CH}_{3}\right)_{3}\right), 1.09$ $1.19\left(12 \mathrm{H}\right.$, mult, $\left.\mathrm{CH}\left(\mathrm{CH}_{3}\right)_{2}\right), 1.30\left(6 \mathrm{H}, \mathrm{d}, \mathrm{CH}\left(\mathrm{CH}_{3}\right)_{2}\right), 3.0\left(4 \mathrm{H}\right.$, mult, $\left.\mathrm{CH}\left(\mathrm{CH}_{3}\right)_{2}\right), 6.64(1 \mathrm{H}, \mathrm{s}$, imidazole backbone $\mathrm{CH}$ ), 7.18-7.36 (6H, mult, $m-\mathrm{CH}+p$-CH). ${ }^{13} \mathbf{C}\left\{{ }^{1} \mathbf{H}\right\}$ NMR (298 K, d8THF) $\delta$ (ppm) $0.2\left(\mathrm{CH}_{2} \mathrm{SiMe}_{3}\right), 3.7\left(\mathrm{Si}\left(\mathrm{CH}_{3}\right)_{3}\right), 23.3\left(\mathrm{CH}\left(\mathrm{CH}_{3}\right)_{2}\right), 25.1\left(\mathrm{CH}\left(\mathrm{CH}_{3}\right)_{2}\right), 25.3$ $\left(\mathrm{CH}\left(\mathrm{CH}_{3}\right)_{2}\right), 26.4\left(\mathrm{CH}\left(\mathrm{CH}_{3}\right)_{2}\right), 28.5\left(\mathrm{CH}\left(\mathrm{CH}_{3}\right)_{2}\right), 28.6\left(\mathrm{CH}\left(\mathrm{CH}_{3}\right)_{2}\right), 123.6(\mathrm{Ar}-\mathrm{CH}), 124.1$ $(\mathrm{Ar}-\mathrm{CH}), 127.9(\mathrm{Ar}-\mathrm{CH}), 128.8(\mathrm{Ar}-\mathrm{CH}), 129.5$ (imidazole backbone $\mathrm{CH}), 139.1$ (Ar- $C$ ), 142.7 (Ar-C), 147.3 (Ar-C), 147.5 (Ar- $C), 155.2$ (C-Ga), $202.8(C:)$.

Synthesis of (THF) $)_{3} \mathrm{~K}\left[: \mathrm{C}\left\{\left[\mathrm{N}\left(2,6-{ }^{i} \mathrm{Pr}_{2} \mathrm{C}_{6} \mathrm{H}_{3}\right)\right]_{2} \mathrm{CHCGa}\left(\mathrm{CH}_{2} \mathrm{SiMe}_{3}\right)_{3}\right\}\right](2)$

Equimolar amounts of $\mathrm{K}\left(\mathrm{CH}_{2} \mathrm{SiMe}_{3}\right)(0.26 \mathrm{~g}, 2 \mathrm{mmol})$ and $\mathrm{IPr}(0.8 \mathrm{~g}, 2 \mathrm{mmol})$ were suspended in hexane $(10 \mathrm{~mL})$ and stirred for $2 \mathrm{~h}$ at room temperature. To the obtained slurry, a hexane solution of $\mathrm{Ga}\left(\mathrm{CH}_{2} \mathrm{SiMe}_{3}\right)_{3}(0.66 \mathrm{~g}, 2 \mathrm{mmol}$ in $10 \mathrm{~mL}$ hexane) was added via cannula and stirred over night at room temperature. The reaction mixture was then concentrated to approximately $5 \mathrm{~mL}$ and $1 \mathrm{~mL}$ of THF was added to afford a straw solution. Overnight storage of the solution at $-30{ }^{\circ} \mathrm{C}$ provided a batch of colourless crystals $(1.48 \mathrm{~g}, 76$ $\%)$. It should be noted that one coordinated THF molecule is lost upon drying in vacuo. Anal. Calcd for $\mathrm{C}_{47} \mathrm{H}_{84} \mathrm{~N}_{2} \mathrm{Si}_{3} \mathrm{KO}_{2} \mathrm{Ga}$ : C, 62.57; H, 9.38; N, 3.10. Found: C, 62.71; H, 9.62; N, 3.45. $\%)$.

${ }^{1} \mathbf{H}$ NMR (298 K, d8-THF) $\boldsymbol{\delta}(\mathbf{p p m})-1.16\left(6 \mathrm{H}, \mathrm{s}, \mathrm{CH}_{2} \mathrm{SiMe}_{3}\right),-0.17\left(27 \mathrm{H}, \mathrm{s}, \mathrm{Si}\left(\mathrm{CH}_{3}\right)_{3}\right), 1.09$ $\left(12 \mathrm{H}, \mathrm{d}, \mathrm{CH}\left(\mathrm{CH}_{3}\right)_{2}\right), 1.17\left(6 \mathrm{H}, \mathrm{d}, \mathrm{CH}\left(\mathrm{CH}_{3}\right)_{2}\right), 1.29\left(6 \mathrm{H}, \mathrm{d}, \mathrm{CH}\left(\mathrm{CH}_{3}\right)_{2}\right), 3.02$ (4H, mult, $\left.\mathrm{CH}\left(\mathrm{CH}_{3}\right)_{2}\right), 6.59(1 \mathrm{H}, \mathrm{s}$, imidazole backbone $\mathrm{CH}), 7.15-7.28(6 \mathrm{H}$, mult, $m-\mathrm{CH}+p-\mathrm{CH})$. ${ }^{13} \mathbf{C}\left\{{ }^{1} \mathbf{H}\right\}$ NMR $\left(298 \mathbf{~ K}\right.$, d8-THF) $\delta(\mathbf{p p m}) 0.2\left(\mathrm{CH}_{2} \mathrm{SiMe}_{3}\right), 3.7\left(\mathrm{Si}\left(\mathrm{CH}_{3}\right)_{3}\right), 23.2\left(\mathrm{CH}\left(\mathrm{CH}_{3}\right)_{2}\right)$, $24.9\left(\mathrm{CH}\left(\mathrm{CH}_{3}\right)_{2}\right), 25.3\left(\mathrm{CH}\left(\mathrm{CH}_{3}\right)_{2}\right), 26.4\left(\mathrm{CH}\left(\mathrm{CH}_{3}\right)_{2}\right), 28.4\left(\mathrm{CH}\left(\mathrm{CH}_{3}\right)_{2}\right), 28.6\left(\mathrm{CH}_{\left.\left(\mathrm{CH}_{3}\right)_{2}\right)}\right)$, $123.2(\mathrm{Ar}-\mathrm{CH}), 123.7(\mathrm{Ar}-\mathrm{CH}), 127.6(\mathrm{Ar}-\mathrm{CH}), 128.2(\mathrm{Ar}-\mathrm{CH}), 128.6$ (imidazole backbone $C H$ ), 140.8 (Ar- $C$ ), 144.3 (Ar-C), 147.3 (Ar-C), 147.4 (Ar- $C$ ), 153.6 (C-Ga), $210.7(C$ :).

Synthesis of $\left[\mathrm{C}_{3} \mathrm{H}_{5} \mathrm{C}\left\{\left[\mathrm{N}\left(2,6-{ }^{i} \mathrm{Pr}_{2} \mathrm{C}_{6} \mathrm{H}_{3}\right)\right]_{2} \mathrm{CHCGa}\left(\mathrm{CH}_{2} \mathrm{SiMe}_{3}\right)_{3}\right\}\right](3)$

To a THF solution of $\mathbf{1}(0.48 \mathrm{~g}, 0.5 \mathrm{mmol}$ in $5 \mathrm{~mL}$ of THF) allyl bromide $(0.06 \mathrm{~g}, 43 \mu \mathrm{L}, 0.5$ mmol) was added inducing precipitation. Obtained suspension was stirred for $1 \mathrm{~h}$ at room 
temperature and then filtered through Celite. Orange filtrate was layered with $3 \mathrm{~mL}$ of hexane and stored at $-33{ }^{\circ} \mathrm{C}$ to afford colourless crystals of title compound (0.16 g, 42\%). Anal. Calcd. for $\mathrm{C}_{42} \mathrm{H}_{73} \mathrm{~N}_{2} \mathrm{Si}_{3} \mathrm{Ga}$ : C, 66.37; H, 9.68; N, 3.69. Found: C, 65.69; H, 9.66; N, 3.84. The NMR analysis is very complex and the reported chemical shifts are for both $\mathrm{CH}=\mathrm{CH}-\mathrm{CH}_{3}$ and $\mathrm{CH}_{2}-\mathrm{CH}=\mathrm{CH}_{2}$ fragments.

${ }^{1} \mathbf{H}$ NMR (298 K, d8 -THF) $\delta$ (ppm) $-0.67\left(6 \mathrm{H}\right.$, mult, $\left.\mathrm{CH}_{2} \mathrm{SiMe}_{3}\right),-0.29$ and $-0.33(27 \mathrm{H}$, s, $\left.\mathrm{Si}\left(\mathrm{CH}_{3}\right)_{3}\right), 0.81-1.46$ (24H, mult, $\left.\mathrm{CH}\left(\mathrm{CH}_{3}\right)_{2}\right), 2.35\left(0.8 \mathrm{H}\right.$, mult, $\left.\mathrm{CH}\left(\mathrm{CH}_{3}\right)_{2}\right), 2.51(1.2 \mathrm{H}$, mult, $\left.\mathrm{CH}\left(\mathrm{CH}_{3}\right)_{2}\right), 2.70\left(0.8 \mathrm{H}\right.$, mult, $\left.\mathrm{CH}\left(\mathrm{CH}_{3}\right)_{2}\right), 2.85\left(1.2 \mathrm{H}\right.$, mult, $\left.\mathrm{CH}\left(\mathrm{CH}_{3}\right)_{2}\right), 2.95(0.5 \mathrm{H}$. d, $\left.\mathrm{CH}=\mathrm{CH}-\mathrm{CH}_{3}\right),[4.12,4.36,4.54,4.67,5.00,5.60]\left(\mathrm{CH}_{2}-\mathrm{CH}=\mathrm{CH}_{2}+\mathrm{CH}=\mathrm{CH}-\mathrm{CH}_{3}\right), 6.88(7 \mathrm{H}$, mult, imidazole backbone $\mathrm{CH}+\mathrm{Ar}-\mathrm{CH}),{ }^{13} \mathrm{C}\left\{{ }^{1} \mathbf{H}\right\}$ NMR (298 K, d8-THF) $\delta(\mathbf{p p m}) 0.3$ $\left(\mathrm{CH}_{2} \mathrm{SiMe}_{3}\right), \quad 3.6 \quad\left(\mathrm{Si}\left(\mathrm{CH}_{3}\right)_{3}\right), \quad 22.6 \quad\left(\mathrm{CH}\left(\mathrm{CH}_{3}\right)_{2}\right), \quad 22.8\left(\mathrm{CH}\left(\mathrm{CH}_{3}\right)_{2}\right), \quad 23.0\left(\mathrm{CH}\left(\mathrm{CH}_{3}\right)_{2}\right)$, 23.7 $\left(\mathrm{CH}\left(\mathrm{CH}_{3}\right)_{2}\right), \quad 23.8\left(\mathrm{CH}\left(\mathrm{CH}_{3}\right)_{2}\right), \quad 23.9\left(\mathrm{CH}\left(\mathrm{CH}_{3}\right)_{2}\right), \quad 24.4\left(\mathrm{CH}\left(\mathrm{CH}_{3}\right)_{2}\right), \quad 24.5\left(\mathrm{CH}\left(\mathrm{CH}_{3}\right)_{2}\right)$, $24.6\left(\mathrm{CH}\left(\mathrm{CH}_{3}\right)_{2}\right), \quad 26.4\left(\mathrm{CH}\left(\mathrm{CH}_{3}\right)_{2}\right), \quad 28.3\left(\mathrm{CH}\left(\mathrm{CH}_{3}\right)_{2}\right), \quad 28.4\left(\mathrm{CH}\left(\mathrm{CH}_{3}\right)_{2}\right), \quad 28.5\left(\mathrm{CH}\left(\mathrm{CH}_{3}\right)_{2}\right)$, 28.7 $\left(\mathrm{CH}\left(\mathrm{CH}_{3}\right)_{2}\right), 30.1 \quad\left(\mathrm{CH}=\mathrm{CH}-\mathrm{CH}_{3}\right),[114.2,115.9,120.3,124.1,124.4,124.6,124.7$, 124.8, $\left.125.1 \mathrm{CH}_{2}-\mathrm{CH}=\mathrm{CH}_{2}+\mathrm{CH}=\mathrm{CH}-\mathrm{CH}_{3}\right],[130.1,130.2,130.5,130.8,130.9,131.2$, 131.4, 131.7 aromatic $C \mathrm{H}+$ imidazole backbone $C \mathrm{H}$ ], [134.1, 134.7, $136.6 \mathrm{Ar}-\mathrm{C}], 141.6$ (C2-C), [143.7, 145.5, 145.6, 145.7 Ar- $C], 163.4$ (C-Ga).

Synthesis of $\left[\mathrm{Me}_{3} \mathrm{SiC}\left\{\left[\mathrm{N}\left(2,6-{ }^{i} \mathrm{Pr}_{2} \mathrm{C}_{6} \mathrm{H}_{3}\right)\right]_{2} \mathrm{CHCGa}\left(\mathrm{CH}_{2} \mathrm{SiMe}_{3}\right)_{3}\right\}\right](4)$

To a THF solution of $2(0.49 \mathrm{~g}, 0.5 \mathrm{mmol}$ in $5 \mathrm{~mL}$ of THF) dried TMSCl $(0.05 \mathrm{~g}, 63 \mu \mathrm{L}, 0.5$ mmol) was inducing precipitation. Obtained suspension was stirred for $1 \mathrm{~h}$ at room temperature and then filtered through Celite. Clear filtrate was layered with $2 \mathrm{~mL}$ of hexane and stored at $-33{ }^{\circ} \mathrm{C}$ to afford colourless crystals of title compound (0.24 g, 61\%). Anal. Calcd for $\mathrm{C}_{42} \mathrm{H}_{77} \mathrm{~N}_{2} \mathrm{Si}_{4} \mathrm{Ga}$ : C, 63.68; H, 9.80; N, 3.54. Found: C, 62.89; H, 9.46; N, 3.60.

${ }^{1}$ H NMR (298 K, C6D6) $\delta$ (ppm) -0.69 (6H, s, $\left.\mathrm{CH}_{2} \mathrm{SiMe}_{3}\right),-0.46$ (9H, s, $\left.\mathrm{SiCH}_{3}\right), 0.33$ (27H, s, $\left.\mathrm{Si}\left(\mathrm{CH}_{3}\right)_{3}\right), 1.03\left(6 \mathrm{H}, \mathrm{d}, \mathrm{CH}\left(\mathrm{CH}_{3}\right)_{2}\right), 1.09\left(12 \mathrm{H}, \mathrm{d}, \mathrm{CH}\left(\mathrm{CH}_{3}\right)_{2}\right), 1.40\left(6 \mathrm{H}, \mathrm{d}, \mathrm{CH}\left(\mathrm{CH}_{3}\right)_{2}\right), 2.40$ $\left(2 \mathrm{H}\right.$, sept, $\left.\mathrm{CH}\left(\mathrm{CH}_{3}\right)_{2}\right), 2.76\left(2 \mathrm{H}\right.$, sept, $\left.\mathrm{CH}\left(\mathrm{CH}_{3}\right)_{2}\right), 6.95(2 \mathrm{H}, \mathrm{d}, \mathrm{Ar}-\mathrm{CH}), 7.04(2 \mathrm{H}, \mathrm{d}, \mathrm{Ar}-$ $\mathrm{CH}$ ), 7.12-7.21 (3H, mult, Ar- $\mathrm{CH}+\mathrm{CH}$ imidazole backbone) ${ }^{13} \mathbf{C}\left\{{ }^{1} \mathbf{H}\right\} \mathbf{N M R}\left(298 \mathbf{K}, \mathbf{d}_{\mathbf{8}}\right.$ THF) $\delta$ (ppm) $-0.5\left(\mathrm{SiCH}_{3}\right), 1.3\left(\mathrm{CH}_{2} \mathrm{SiMe}_{3}\right), 3.7\left(\mathrm{Si}\left(\mathrm{CH}_{3}\right)_{3}\right), 21.6\left(\mathrm{CH}\left(\mathrm{CH}_{3}\right)_{2}\right), 24.3$ $\left(\mathrm{CH}\left(\mathrm{CH}_{3}\right)_{2}\right), 25.1\left(\mathrm{CH}\left(\mathrm{CH}_{3}\right)_{2}\right), 28.0\left(\mathrm{CH}\left(\mathrm{CH}_{3}\right)_{2}\right), 28.5\left(\mathrm{CH}\left(\mathrm{CH}_{3}\right)_{2}\right), 28.9\left(\mathrm{CH}\left(\mathrm{CH}_{3}\right)_{2}\right), 124.2$ 
(Ar- $\mathrm{CH}), 124.4(\mathrm{Ar}-\mathrm{CH}), 130.5(\mathrm{Ar}-\mathrm{CH}), 131.4(\mathrm{Ar}-\mathrm{CH}), 133.1(\mathrm{Ar}-\mathrm{C}), 135.3$ (imidazole backbone $C H$ ), 137.3 (Ar- $C$ ), 145.3 (Ar- $C$ ), 146.5 (Ar- $C$ ), 148.8 ( $C$-SiMe 3 ), 167.6 ( $C$-Ga).

\section{Representative reaction of alkali-metal gallate with IPr}

To a hexane suspension of $\mathrm{KGaR}_{4}\left(\mathrm{R}=\mathrm{CH}_{2} \mathrm{SiMe}_{3}\right)$ an equivalent of IPr was added via solid addition tube and the obtained suspension stirred at room temperature for $1 \mathrm{~h}$. The suspension was gently heated until the solution was obtained which upon cooling produced colourless crystals. The multinuclear NMR spectroscopic analysis of crystals reveals no deprotonation of the backbone and suggests formation of ligand separated potassium gallate $\left[\left\{\mathrm{K}(\mathrm{IPr})_{2}\right\}^{+}\left\{\mathrm{Ga}\left(\mathrm{CH}_{2} \mathrm{SiMe}_{3}\right)_{4}\right\}^{-}\right]$.

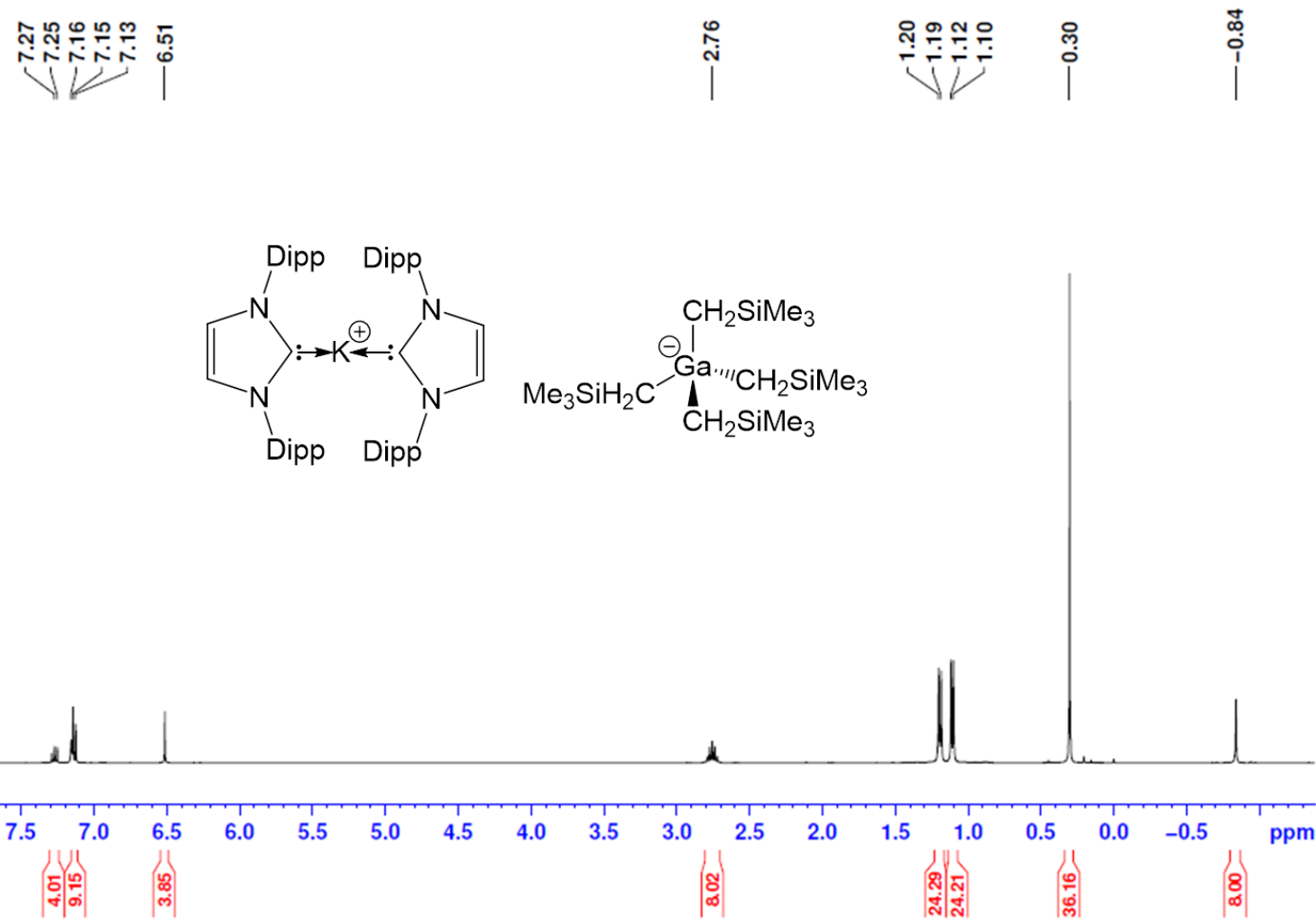

Figure S1: ${ }^{1} \mathrm{H}$ NMR spectrum of $\left[\left\{\mathrm{K}(\mathrm{IPr})_{2}\right\}^{+}\left\{\mathrm{Ga}\left(\mathrm{CH}_{2} \mathrm{SiMe}_{3}\right)_{4}\right\}^{-}\right]$in $\mathrm{C}_{6} \mathrm{D}_{6}$. 




Figure S2: ${ }^{13} \mathrm{C}$ NMR spectrum of $\left[\left\{\mathrm{K}(\operatorname{IPr})_{2}\right\}^{+}\left\{\mathrm{Ga}\left(\mathrm{CH}_{2} \mathrm{SiMe}_{3}\right)_{4}\right\}^{-}\right]$in $\mathrm{C}_{6} \mathrm{D}_{6}$.

\section{NMR spectra}




Figure S3: ${ }^{1} \mathrm{H}$ NMR spectrum of $\mathbf{1}$ in $\mathrm{C}_{6} \mathrm{D}_{6}$.

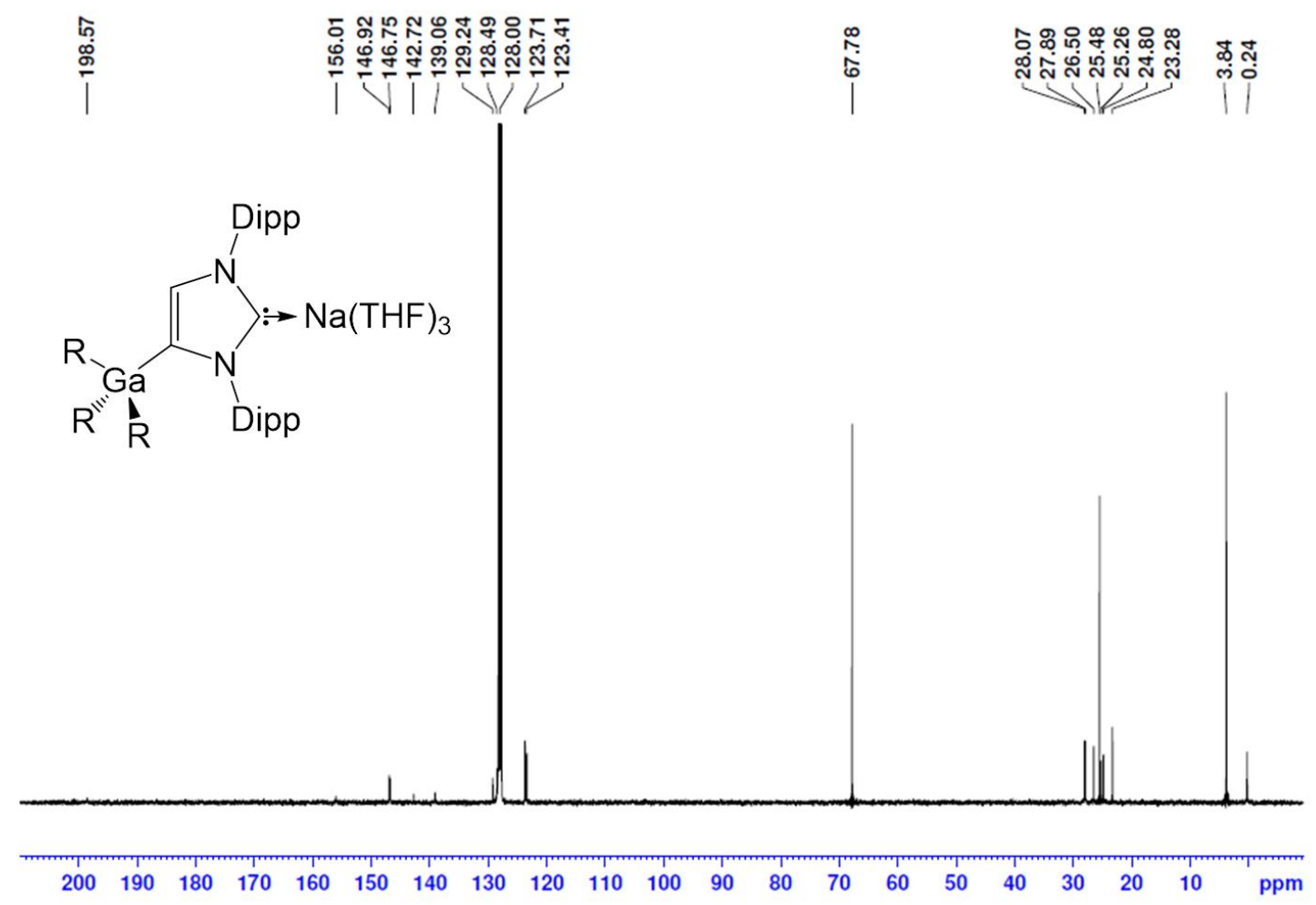

Figure S4: ${ }^{13} \mathrm{C}$ NMR spectrum of $\mathbf{1}$ in $\mathrm{C}_{6} \mathrm{D}_{6}$.

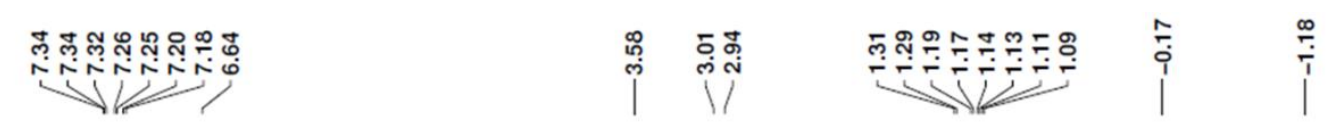



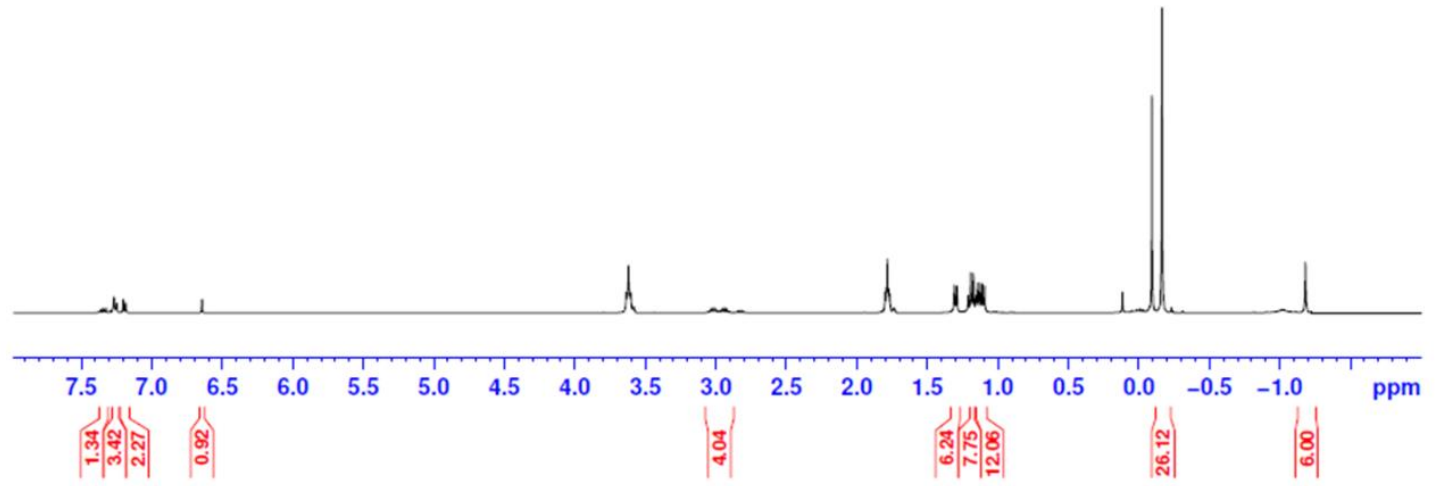

Figure S5: ${ }^{1} \mathrm{H}$ NMR spectrum of $\mathbf{1}$ in $\mathrm{d}_{8}$-THF. 
సิ่

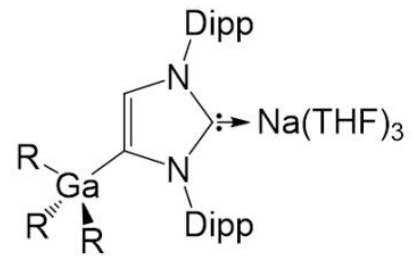

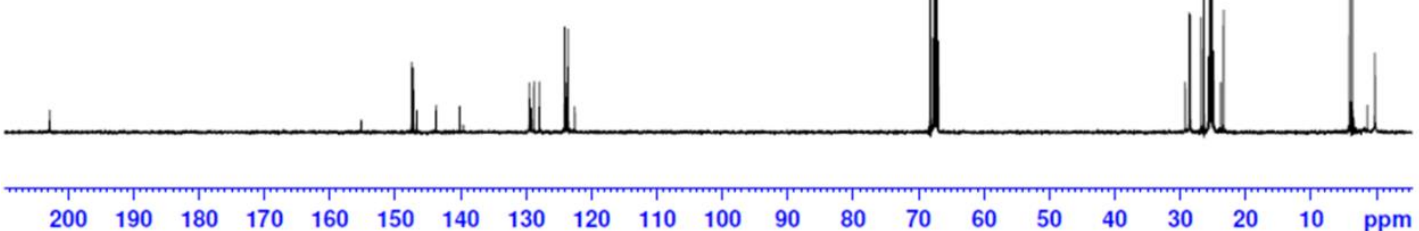

Figure S6: ${ }^{13} \mathrm{C}$ NMR spectrum of $\mathbf{1}$ in $\mathrm{d}_{8}$-THF.

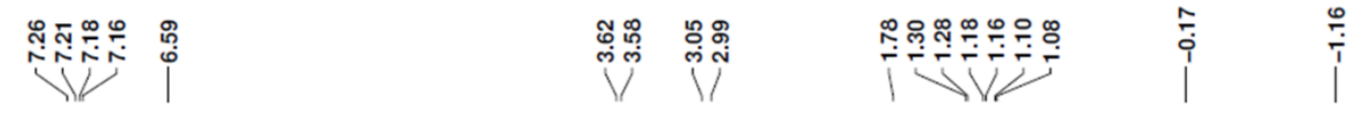

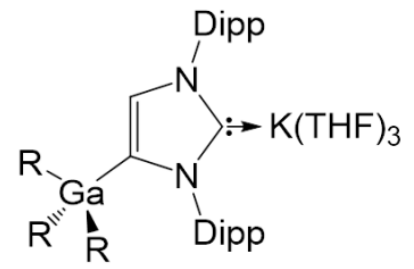

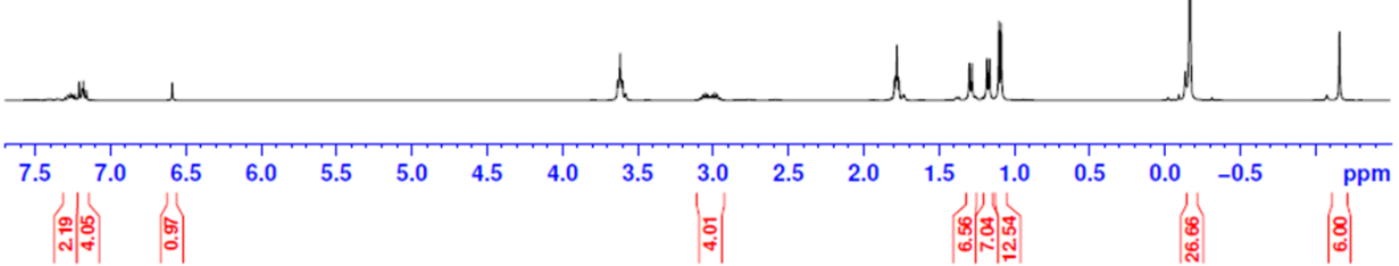

Figure S7: ${ }^{1} \mathrm{H}$ NMR spectrum of $\mathbf{2}$ in $\mathrm{d}_{8}$-THF. 


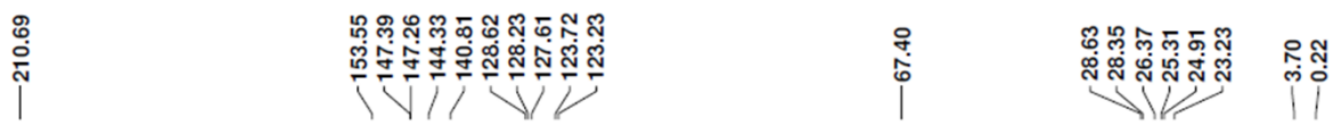

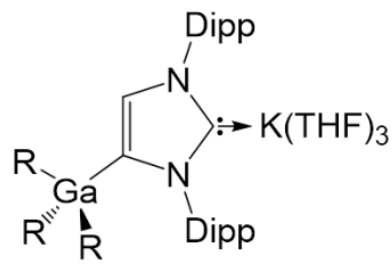

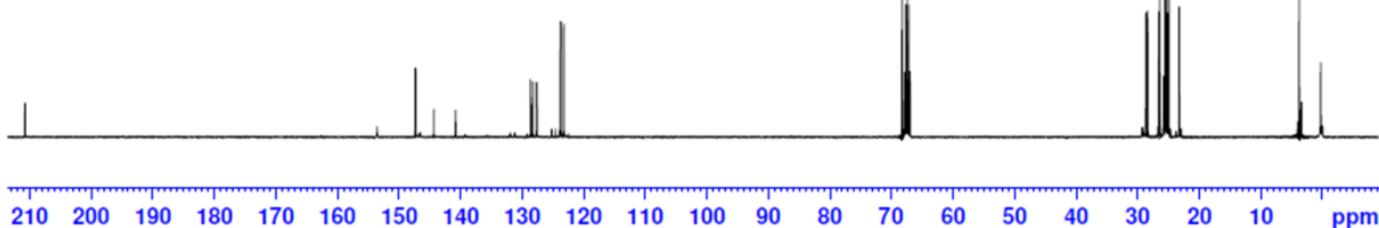

Figure S8: ${ }^{13} \mathrm{C}$ NMR spectrum of 2 in $\mathrm{d}_{8}$-THF.



Figure S9: ${ }^{1} \mathrm{H}$ NMR spectrum of 3 in $\mathrm{C}_{6} \mathrm{D}_{6}$. 

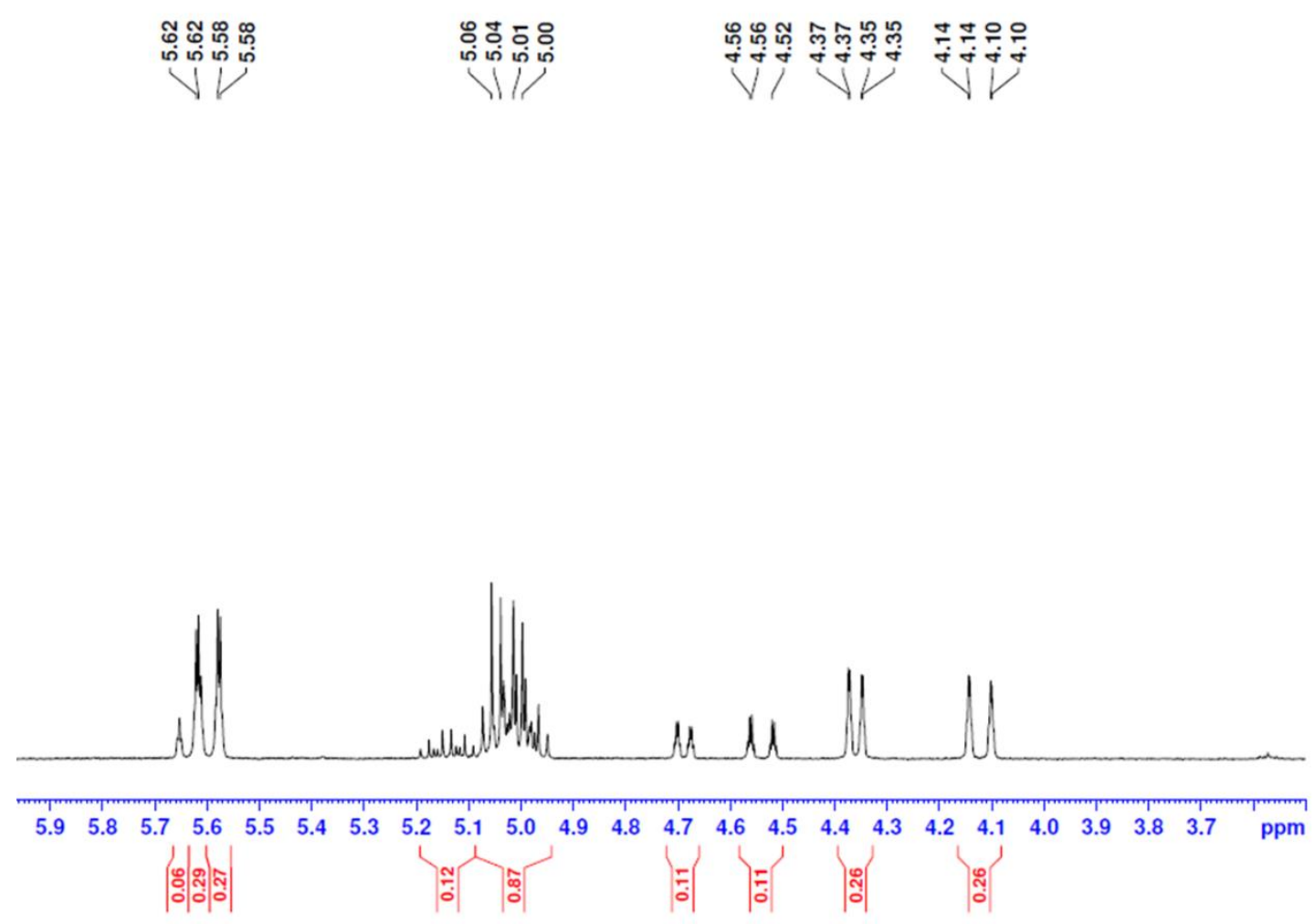

Figure S10: Partial ${ }^{1} \mathrm{H}$ NMR spectrum of $\mathbf{3}$ (6 ppm -3.5 ppm region) in $\mathrm{C}_{6} \mathrm{D}_{6}$.
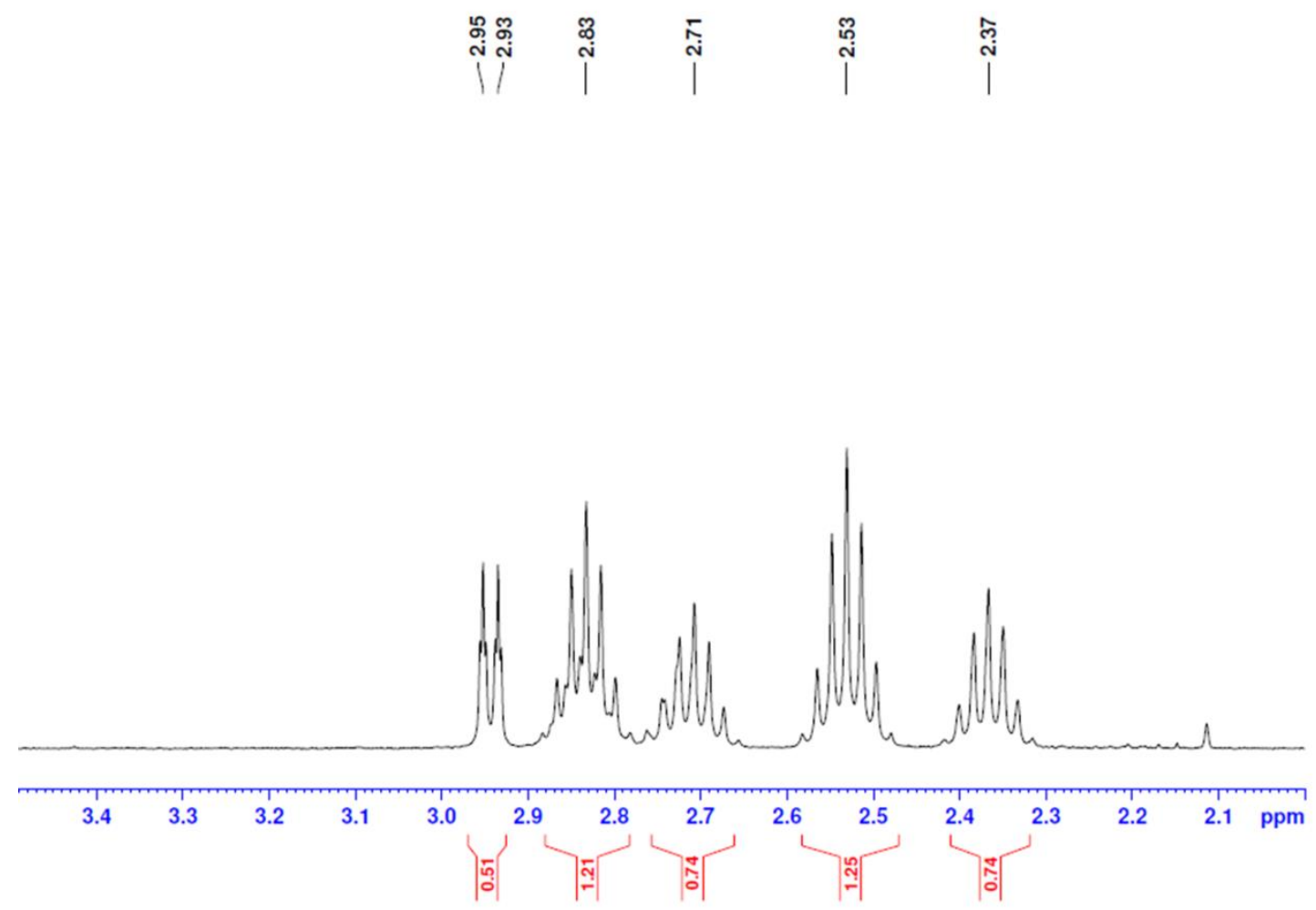

Figure S11: Partial ${ }^{1} \mathrm{H}$ NMR spectrum of $\mathbf{3}$ (3.5 ppm -2 ppm region) in $\mathrm{C}_{6} \mathrm{D}_{6}$. 

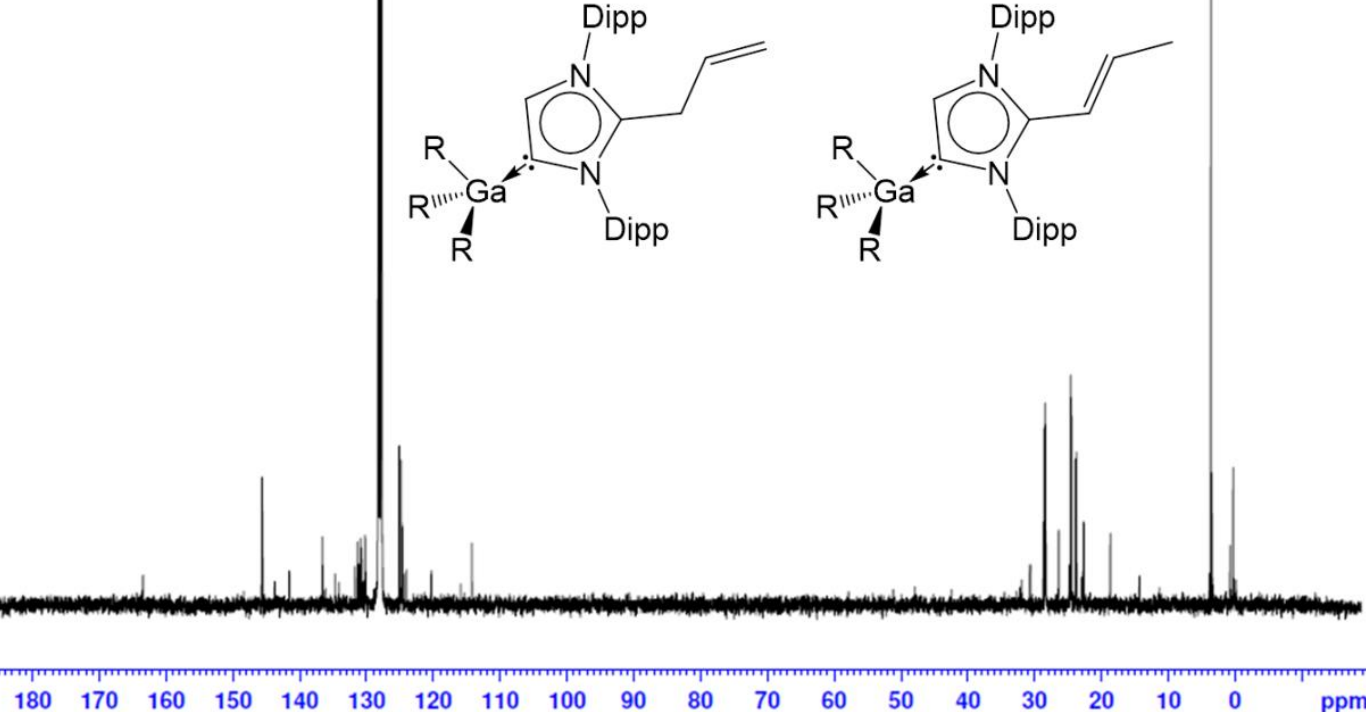

Figure S12: ${ }^{13} \mathrm{C}$ NMR spectrum of 3 in $\mathrm{C}_{6} \mathrm{D}_{6}$.



Figure S13: High field region of ${ }^{13} \mathrm{C}$ NMR spectrum of 3 in $\mathrm{C}_{6} \mathrm{D}_{6}$. 


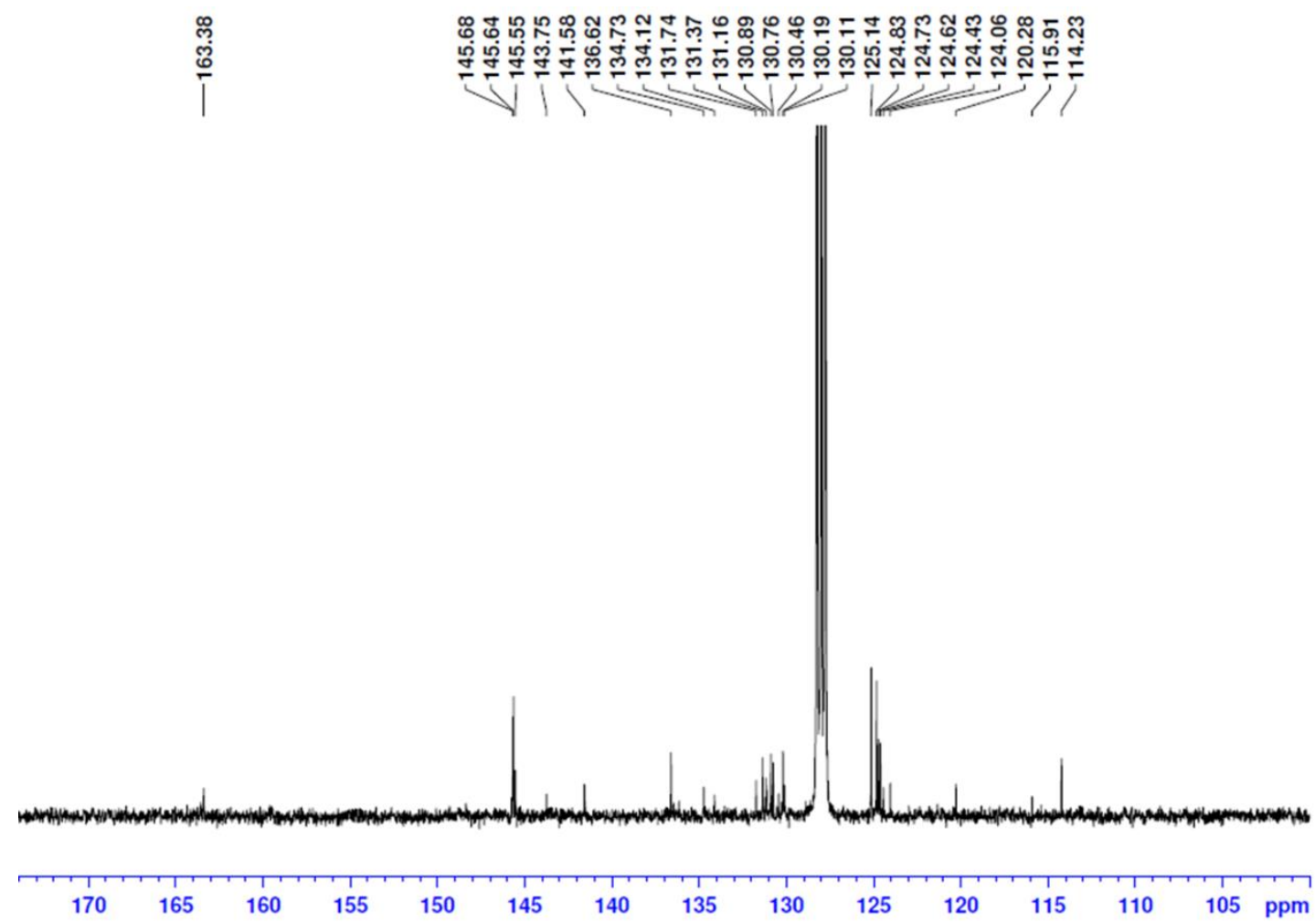

Figure S14: Low field region of ${ }^{13} \mathrm{C}$ NMR spectrum of 3 in $\mathrm{C}_{6} \mathrm{D}_{6}$.

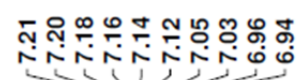



Figure S15: ${ }^{1} \mathrm{H}$ NMR spectrum of 4 in $\mathrm{C}_{6} \mathrm{D}_{6}$. 


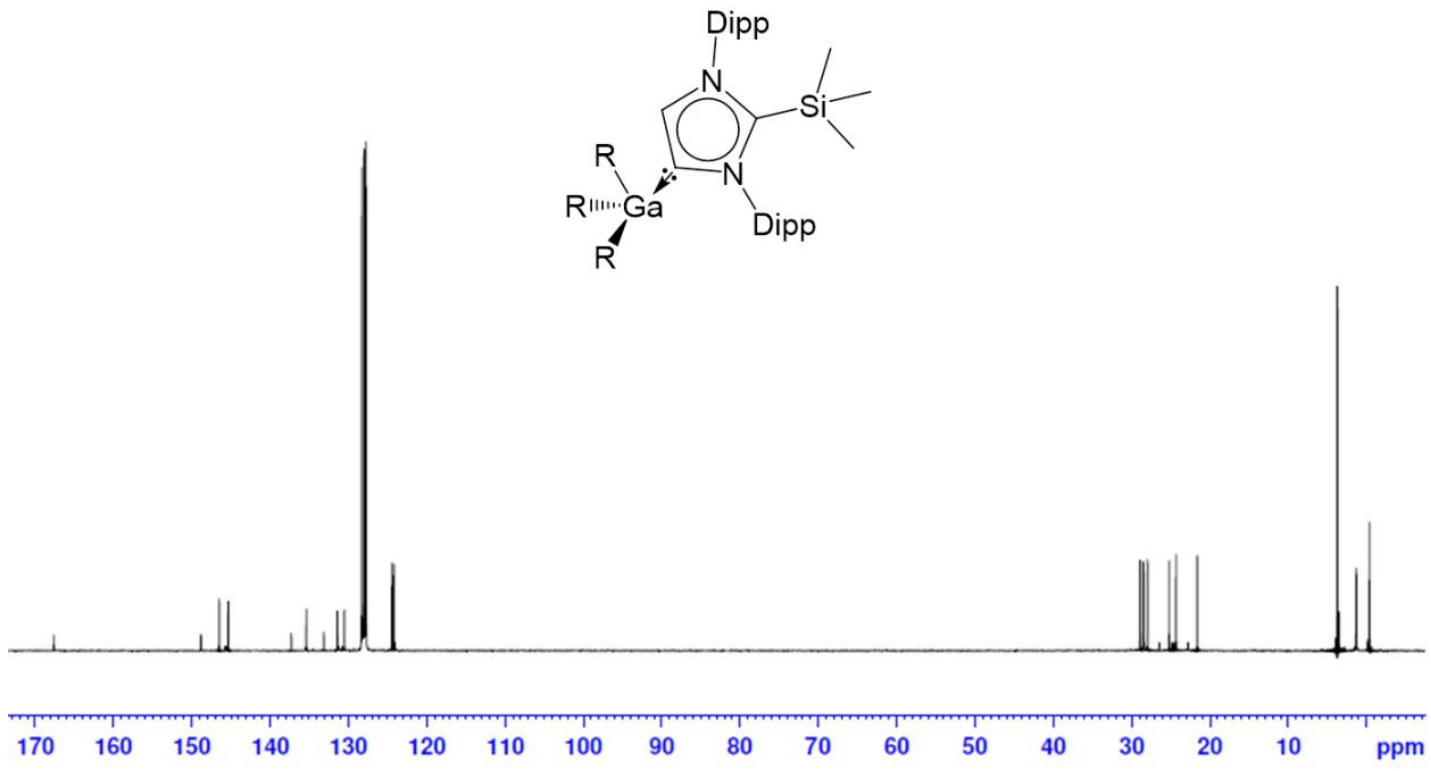

Figure S16: ${ }^{13} \mathrm{C}$ NMR spectrum of 4 in $\mathrm{C}_{6} \mathrm{D}_{6}$. 